\title{
Location Based Tracking System for Emergency Services
}

\author{
T. Swathi \\ Aurora's Technological and Reaserch Institute \\ Uppal, Hyderabad \\ India
}

\author{
B.S. Malleswari \\ Aurora's Technological and Research Institute \\ Uppal, Hyderabad \\ India.
}

\begin{abstract}
Transmitting the geo-location information of a target via wireless networks is effective when both the target and the tracker are within Wi-Fi coverage area; the 802.11 wireless networks are not always accessible. When the target or the tracker is unable to access Wi-Fi, it is impossible to perform location tracking. Therefore, SMS is a relatively more reliable and flexible solution because of its widespread use. In this system, a device is equipped with a global system for mobile communications (GSM) modem and a GPS unit. It transmits short messages containing its GPS coordinates to the server at 30-s intervals. Although transmitting the geo-location information of a target via wireless networks is effective when both the target and the tracker are within Wi-Fi coverage area, the 802.11 wireless networks are not always accessible. When the target or the tracker is unable to access Wi-Fi, it is impossible to perform location tracking. In this System, a novel method called location-based delivery (LBD), which combines the short message service (SMS) and global position system (GPS). LBD reduces the number of short message transmissions while maintaining the location tracking accuracy within the acceptable range. The proposed approach, LBD, consists of three primary features: Short message format, location prediction, and dynamic threshold. The defined short message format is proprietary.

Key Words: Short Message Service (SMS), Location Tracking, Mobile Phones, Prediction Algorithms, Global Positioning System (GPS).
\end{abstract}

\section{INTRODUCTION}

Location based tracking and handling the devices is based on the global position system (GPS) is common in the growing world, and therefore, several location tracking applications have been developed, including continuous location based transport, system or vehicle based intelligent transport, monitoring vehicles, tracking elders, children's and women employees for their safety reasons or to prevent them from the being lost. The GPS is mainly used to obtain geographical location of the object (e.g., a transmitter devices or mobile devices). However, most of the abovecitedworks used either an 802.11 wireless network or the short messageservice (SMS) to transmit the location information of a targetto a tracker. Real time tracking system is majorly used for care management applications for

children and mentally challenged people; the main aim of the system is to transfer the location and position of the objective to the mobile device to a central GPS application server through the 802.11 wireless networks. This application allows the server to simultaneously monitor multiple targets (e.g., elders or children), this is in line with Lee et al.Further, Choi et al. assumed that the location information of a targets transmitted through wireless networks. Their work focused on proposing a geolocation update scheme to decrease the update. Frequency. Lita et al. proposed an automobile localization system by using SMS.The proposed system, which is interconnectedwith the car alarm system, transmits alerts to theowner's mobile phone in the event of a car theft (e.g., activationof the car alarm, starting of the engine) or provides informationfor monitoring adolescent drivers (e.g., exceeding the speedlimit or leaving a specific area). Hameed et al. proposed a carmonitoring and tracking system that uses both SMS and GPSto prevent car theft. Anderson et al. proposed a transportationinformation system. In this system, a hardware device called Star Box, which is equipped with a global system for mobile communications (GSM) modem and a GPS unit, is installed in a vehicle to track the vehicle's location. Star Box transmits short messages containing its GPS coordinates to the server at30$\mathrm{s}$ intervals. The users can send short messages to the server to determine the expected arrival time of buses at their locations. Although transmitting the geolocation information of a targetvia wireless networks is effective when both the target and the tracker are within Wi-Fi coverage area, the 802.11 wireless networksare not always accessible. When the target or the trackeris unable to access Wi-Fi, it is impossible to perform locationtracking. Therefore, SMS is a relatively more reliable and flexiblesolution because of its widespread use (i.e., wellstructuredworldwide) [6], [8]. However, SMS is a user-pay service.

The objective of this study is to minimize the transmission cost ofa tracking system by minimizing the number of SMS transmissionswhile maintaining the location tracking accuracy.

In this paper, a novel method called location-based delivery (LBD), which combines SMS and GPS, is proposed, and further, a realistic system to perform precise location trackingis developed. LBD mainly applies the following two proposedtechniques: Location prediction and dynamic threshold. Locationprediction is performed by using the current location, movingspeed, and bearing of the target to predict its next location. When the distance between the predicted location and theactual location exceeds a certain 
threshold, the target transmitsa shortmessage to the tracker to update its current location. Thedynamic threshold maintains the location tracking accuracy andnumber of short messages on the basis of the moving speed ofthe target.The simulations performed to test the performance of LBD show that compared with other related works; the proposed LBD minimizes the number of short message transmissions while maintaining the location prediction accuracy within the acceptable range.

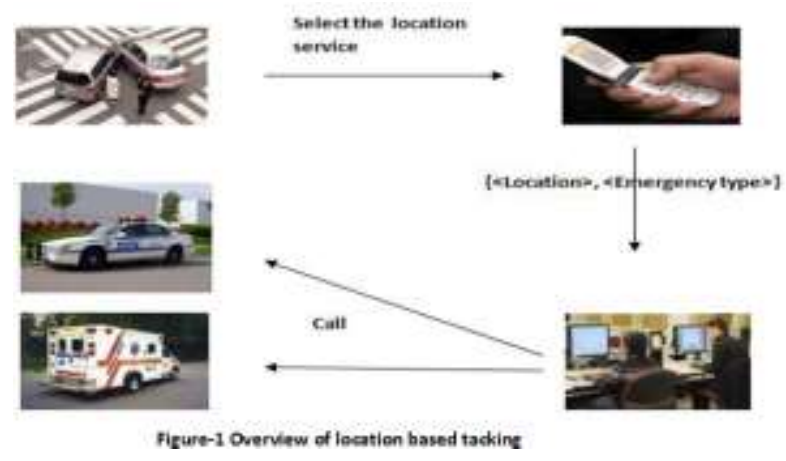

Figure-1 Overview of location based tracking.

\section{SHORT MESSAGE SERVICE}

SMS is a text messaging service component of phone, web, or mobile communication systems, using standardized communications protocols that allow the exchange of short text messages between fixed line or mobile phone devices. SMS text messaging is the most widely used data application in the world, with 3.6 billion active users, or $78 \%$ of all mobile phone subscribers. A short message is transmitted from the mobile station (MS) to the GSM base station (BTS) through a wireless link and is received in the backbone network of the service provider. The mobile switch center (MSC), home location register (HLR), and visitor location register (VLR) determine the appropriate short message service center (SMSC), which processes the message by applying the "store and forward" mechanism.

The term SMS is used as a synonym for all types of short text messaging as well as the user activity itself in many parts of the world. SMS is also being used as a form of direct marketing known as SMS marketing. SMS as used on modern handsets originated from radio telegraphy in radio memo pagers using standardized phone protocols and later defined as part of the GSM (Global System for Mobile Positioning) series of standards in 1985 as a means of sending messages of up to 160 characters, to and from GSM mobile handsets. Since then, support for the service has expanded to include other mobile technologies such as ANSI CDMA networks and digital AMPs, as well as satellite and landline networks. Most SMS messages are mobile-to-mobile text messages though the standard supports other types of broadcast messaging as well.

\section{LOCATION BASED DELIVERY}

Location based services (LBS) a novel class of computer application, which combines the short message services (SMS) and global position system (GPS). As such LBS is an informative service and number of uses in social networking today as an entertainment service, which is accessible with mobile devices through the mobile network and which uses information on the geographical position of the mobile device.

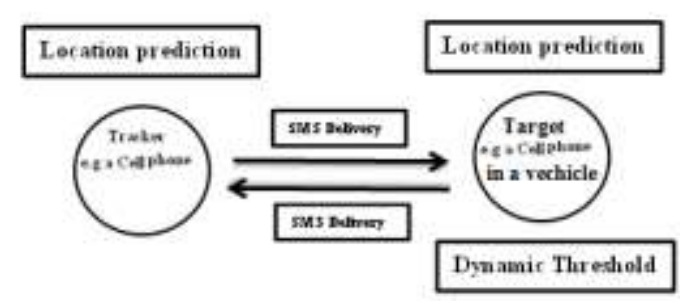

Figure-2 Structure of the LBD systeem.

The proposed approach, LBD, consists of three primary features: Shortmessage format, location prediction, and dynamic threshold. The defined short message format is proprietary. Location prediction isperformed by using the current location, moving speed, and bearing of the target to predict its next location. When the distance between the predicted location and the actual location exceeds a certain threshold, the target transmits a short message to the trackerto update its current location. The threshold is dynamically adjusted to maintain the location tracking accuracy and the numberof short messages on the basis of themoving speed of the target. It satisfactorily maintains the location tracking accuracy with relatively fewer messages.The threshold is dynamically adjusted to maintain the location tracking accuracy.

\section{CONCLUSION:}

In this System, a novel method called location-based delivery (LBD), which combines the short message service (SMS) and global position system (GPS). LBD reduces the number of short message transmissions while maintaining the location tracking accuracy within the acceptable range. The proposed approach, LBD, consists of three primary features: Short message format, location prediction, and dynamic threshold. The defined short message format is proprietary. Location prediction is performed by using the current location, moving speed, and bearing of the target to predict its next location. When the distance between the predicted location and the actual location exceeds a certain threshold, the target transmits a short message to the trackerto update its current location. The threshold is dynamically adjusted to maintain the location tracking accuracy and the number of short messages on the basis of the moving speed of the target. 
International Journal of Computer Applications Technology and Research Volume 3- Issue 12, 836 - 838, 2014, ISSN:- 2319-8656

\section{REFERENCES}

[1] H. H. Lee, I. K. Park, and K. S. Hong, "Design and implementation ofa mobile devices-based real-time location tracking," in Proc. UBICOMM, 2008, pp. 178183.

[2] Z. Tian, J. Yang, and J. Zhang, "Location-based services applied to aelectric wheelchair based on the GPS and GSM networks," in Proc. ISA,2009, pp. 1-4.

[3] I. Lita, I. B. Cioc, and D. A. Visan, "A new approach of automobile localizationsystem using GPS and GSM/GPRS transmission," in Proc. ISSE,2006, pp. 115-119.

[4] P. Perugu, "An innovative method using GPS tracking, WINS technologiesfor border security and tracking of vehicles," in Proc. RSTSCC, 2010, pp. 130-133.

[5] S. A. Hameed, O. Khalifa, M. Ershad, F. Zahudi, B. Sheyaa, and W.Asender, "Car monitoring, alerting, and tracking model: Enhancementwith mobility and database facilities," in Proc. ICCCE, 2010, pp. 1-5.

[6] R. E. Anderson, A. Poon, C. Lustig, W. Brunette, G. Borriello, and B. E.Kolko, "Building a transportation information system using only GPS andbasic SMS infrastructure," in Proc. ICTD, 2009, pp. 233-242.

[7] W. J. Choi and S. Tekinay, "Location-based services for next-generationwireless mobile networks," in Proc. IEEE VTC, 2003, pp. 1988-1992.

[8] R. E. Anderson, W. Brunette, E. Johnson, C. Lustig, A. Poon, C. Putnam,

O. Salihbaeva, B. E. Kolko, and G. Borrielllo, "Experiences with atransportation information system that uses only GPS and SMS," in Proc.ICTD, 2010.

[9] A. Civilis, C. S. Jensen, and S. Pakalnis, "Techniques for efficient roadnetwork-based tracking of moving objects," IEEE Trans. Knowl.DataEng., vol. 17, no. 5, pp. 698-712, 2005.

[10] M. Zahaby, P. Gaonjur, and S. Farajian, "Location tracking in GPS usingKalman filter through SMS," in Proc. IEEE EUROCON, 2009, pp. 1707-1711.

[11] A. Civilis, C. S. Jensen, J. Nenortaite, and S. Pakalnis, "Efficient trackingof moving objects with precision guarantees," in Proc. MOBIQUITOUS,2004, pp. 164-173.

[12] Y. Y. Xiao, H. Zhang, and H. Y. Wang, "Location prediction for trackingmoving objects based on grey theory," in Proc. FSKD, 2007, pp. 390-394.

[13] P. H. Tseng, K. T. Feng, Y. C. Lin, and C. L. Chen, "Wireless locationtracking algorithms for environments with insufficient signal sources,"IEEE Trans. Mobile Comput., vol. 8, no. 12, pp. 1676-1689, 2009.

[14] R. Bajaj, S. L. Ranaweera, and D. P. Agrawal, "GPS: Location-trackingtechnology," Computer, vol. 35, no. 4, pp. 92-94, 2002.

[15] Movable Type Scripts. (2012 June).[Online]. Available: http://www.mova

ble-type.co.uk/scripts/latlong.html 